\title{
Transition from IVR limited vibrational energy transport to bulk heat transport
}

\author{
Schade, Marco ; Hamm, Peter
}

\begin{abstract}
In a previous paper (J. Chem. Phys. 131, 044511 (2009)), it has been shown that on ultrashort length and time scales, the speed of vibrational energy transport along a molecular chain is limited by intrasite vibrational relaxation rather than the actual inter site propagation. However, since intrasite vibrational relaxation is length independent, the inter site propagation rate is expected to become ratelimiting at some length scale, where propagation approaches the bulk limit. In the present paper, we investigate the transition between both regimes. The response of different types of modes may be very different at early times, depending on how much they contribute directly to energy transport. Surprisingly though, when averaging the energy content over all vibrational modes of the various chain sites, the complexity of the intrasite vibrational relaxation process is completely hidden so that energy transport on the nanoscale can be described by an effective propagation rate, that equals the bulk value, even at short times.
\end{abstract}

DOI: https://doi.org/10.1016/j.chemphys.2011.11.018

Posted at the Zurich Open Repository and Archive, University of Zurich

ZORA URL: https://doi.org/10.5167/uzh-64647

Journal Article

Accepted Version

Originally published at:

Schade, Marco; Hamm, Peter (2012). Transition from IVR limited vibrational energy transport to bulk heat transport. Chemical Physics, 393(1):46-50.

DOI: https://doi.org/10.1016/j.chemphys.2011.11.018 


\title{
Transition from IVR limited vibrational energy transport to bulk heat transport
}

\author{
Marco Schade and Peter Hamm \\ Physikalisch-Chemisches Institut, Universität Zürich, Winterthurerstr. 190, CH- \\ 8057 Zürich, Switzerland
}

\begin{abstract}
In a previous paper (J. Chem. Phys. 131, 044511 (2009)), it has been shown that on ultrashort length and time scales, the speed of vibrational energy transport along a molecular chain is limited by intrasite vibrational relaxation rather than the actual intersite propagation. However, since intrasite vibrational relaxation is length independent, the intersite propagation rate is expected to become rate-limiting at some length scale, where propagation approaches the bulk limit. In the present paper, we investigate the transition between both regimes. The response of different types of modes may be very different at early times, depending on how much they contribute directly to energy transport. Surprisingly though, when averaging the energy content over all vibrational modes of the various chain sites, the complexity of the intrasite vibrational relaxation process is completely hidden so that energy transport on the nanoscale can be described by an effective propagation rate, that equals the bulk value, even at short times.
\end{abstract}

\section{Introduction}

Heat transport in nanoscale objects [1] has reached considerable interest, both in low-dimensional structures like nanotube thermal rectifiers [2], anharmonic molecular junctions [3], longchain hydrocarbon molecules [4] and bridged azulene-anthracene 
compounds [5], but also in more complicated structures reaching from single amino acids [6,7], small molecules in solution [8], phospholipid bilayer liposomes [9], reverse micelles [10] to real proteins [11-14]. In all cases, large temperature gradients are generated over length scales of a few chemical bonds and vibrational excess energy is dissipated on a few picosecond timescale. Realistic molecular chains do not just consist of simple $\mathrm{C}-\mathrm{C}$ bonds, but contain more complicated molecular repeat units (e.g. amino acids in a protein) with many intra-site degrees of freedom. Intramolecular vibrational energy redistribution (IVR) between these degrees of freedom is a process that typically takes place on a $1-10$ ps timescale [15-17]. Consequently, vibrational energy does not necessarily thermalize within a given site on the timescale of transport, so that familiar concepts like "temperature" or "heat transport" break down [4]. Furthermore, on the more practical side, it is no longer clear how to measure the "temperature", i.e., how to experimentally implement a good "thermometer" on the microscopic scale [18].

To gain a microscopic understanding of vibrational energy transport phenomena on this sort of length and time scales, we have recently introduced a new experimental concept, which we applied to short 310 -helical peptides: vibrational excess energy is deposited at one position of the peptide by various kinds of local heating mechanisms, i.e., the ultrafast electronic relaxation of an azobenzene-moiety $[19,20]$, vibrational relaxation of $\mathrm{C}-\mathrm{D}$ modes [21], or the relaxation of the plasmon resonance in gold nanoparticles [22]. The subsequent flow of vibrational energy through the helix is detected with subpicosecond time resolution by employing vibrational probes at various distances from the heating source as local thermometers. For this purpose, certain $\mathrm{C}=\mathrm{O}$ vibrations are singled out from the main amide I band by site-selective isotope labeling. Upon heating the immediate surrounding of such a localized mode, the latter shifts in frequency and broadens due to anharmonic couplings [23].

Independent of the heating mechanism, the apparent heat dif- 
fusivity we observed was consistently $\approx 2 \AA^{2} \mathrm{ps}^{-1}$, which is significantly slower than values known for bulk-materials. For instance, the heat diffusivity is $14 \AA^{2} \mathrm{ps}^{-1}$ for bulk water [24] and 10-15 $\AA^{2} \mathrm{ps}^{-1}$ for proteins [25]. Overall, the heat diffusivity varies only little with material. Accompanying non-equilibrium molecular dynamics (MD) simulations, mimicking the experiment in atomistic detail, revealed a heat diffusivity that was five time faster than observed experimentally [19]. The MD result is consistent with other theoretical works [26-35] and also agrees with experimental bulk values.

To resolve the discrepancies between our experiments and the accompanying MD simulations [19], we have recently introduced a small toy model for simulating vibrational energy transport along linear chains in the presence of intrasite IVR [36]. The model was designed to mimic the normal mode distribution found in proteins (which is similar like in one-dimensional glasses), in which only a relatively small subset of low-frequency modes tend to delocalize over large distances and as such dominate vibrational energy transport [37-39,26-29,40]. The higher-frequency modes, in contrast, are largely localized at individual sites. We could show that on the given ultrashort length and time scales, intra-site IVR rather than the actual transport along the chain is the rate limiting step, which significantly slows down the apparent propagation speed. However, since intra-site IVR is chain-length independent, the intersite propagation rate is expected to become rate limiting on some larger length scales. Due to the high computational costs of the quantum-mechanical simulations [36], we could not extend the system to length scales on which the expected transition between the intra-site- and inter-site-dominated regime occurs. In the present paper, we revisit the model and treat it on a much simpler level, which can be applied to much longer molecular chains, in order to closer investigate this transition. 


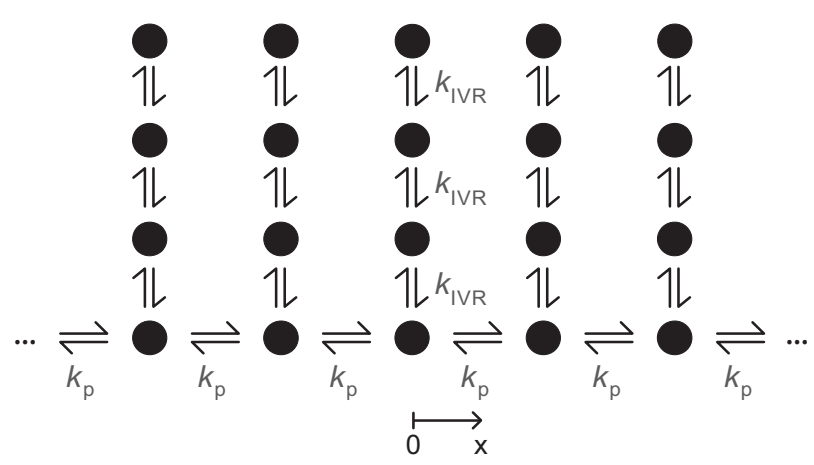

Fig. 1. One-dimensional chain with $n$ sites and $m=4$ modes per site. The transporting mode exchanges vibrational energy with its nearest neighbors at a propagation rate $k_{\mathrm{p}}$. At each site, three ("parking") modes mimic intra-site IVR with rate $k_{\mathrm{IVR}}$. The distance between two adjacent sites is $\Delta x=1$.

\section{Model}

Along the lines of our previous paper [36], a one-dimensional (1D) chain is defined, each consisting of one transporting mode and a set of localized ("parking") modes, as depicted in Fig. 1. The distance between two adjacent chain sites is chosen as $\Delta x=1$. The transporting mode is coupled to its nearest neighbors with a propagation rate $k_{\mathrm{p}}$. The other modes are localized, and can only exchange energy within the respective site at rate $k_{\mathrm{IVR}}$ (intrasite IVR). For simplicity, we choose a cascade-like connectivity of the localized states (like in Ref. [36]) with identical forward and backward rates, but, as shown analytically in the Appendix, the essential result of the paper also holds for much more complex distributions of modes within each site and for arbitrary rates. The dynamics of such a system can be described by set of coupled linear differential equations. As a nonequilibrium initial condition, the chain is locally heated at one single site. Energy on the heating site is deposited in a locally thermalized manner, however with a temperature that is elevated compared to the rest of the chain (as shown in the Appendix, this initial condition is crucial for some of the results of the paper). To avoid boundary effects, the central site of the chain is heated, and the length of the chain is chosen sufficiently large. 


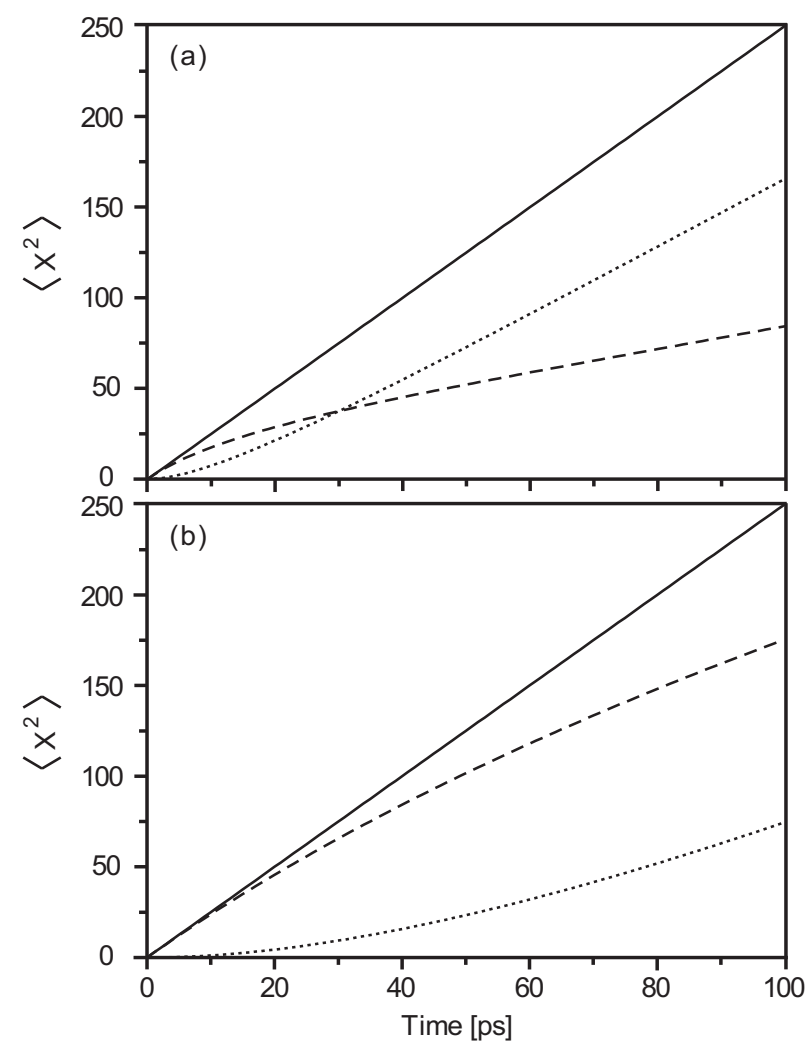

Fig. 2. Mean square displacement of the energy $\left\langle x^{2}\right\rangle$ as a function of time $t$. The presented results were obtained for the model shown in Fig. 1 with a propagation rate $k_{\mathrm{p}}=(0.2 \mathrm{ps})^{-1}$ and (a) a fast IVR rate of $k_{\mathrm{IVR}}=(10 \mathrm{ps})^{-1}$ or (b) a slow IVR rate of $k_{\mathrm{IVR}}=(100 \mathrm{ps})^{-1}$. Shown is the mean square displacement of the total vibrational energy, summed up over all modes of a site $\left\langle x_{\text {site }}^{2}\right\rangle$ (solid lines), and that broken down into the contributions of the transporting modes $\left\langle x_{\text {trans }}^{2}\right\rangle$ (dashed lines) as well as the parking modes $\left\langle x_{\text {park }}^{2}\right\rangle$ (dotted lines).

\section{Results}

The time-dependence of the mean square displacement $\left\langle x^{2}\right\rangle$ is commonly used to assess whether a process is diffusive, in which case it increases linearly with time

$$
\left\langle x^{2}\right\rangle=2 D t
$$

$D$ is the diffusion constant. Fig. 2 shows the mean square displacement of the total vibrational energy, summed over all modes of a site $\left\langle x_{\text {site }}^{2}\right\rangle$ (solid lines), and that broken down into the contributions of the transporting modes $\left\langle x_{\text {trans }}^{2}\right\rangle$ (dashed lines) as well as the localized parking modes $\left\langle x_{\text {park }}^{2}\right\rangle$ (dotted lines). For very 
early times, only vibrational energy that is initially deposited in the transporting mode can contribute to energy diffusion. The contribution of the parking modes, in contrast, initially is low because energy first has to dissipate down the cascade by IVR into the transporting mode. Likewise, once energy arrives at another site, it has to dissipate back into the corresponding parking modes, again with rate $k_{\mathrm{IVR}}$. Hence, the parking modes start to contribute to the overall transport only after a time given by $k_{\mathrm{IVR}}$. This becomes evident from the comparison of the results obtained for a fast IVR rate $\left(k_{\mathrm{IVR}}=(10 \mathrm{ps})^{-1}\right.$, Fig. 2a) and a slow IVR rate $\left(k_{\mathrm{IVR}}=(100 \mathrm{ps})^{-1}\right.$, Fig. $\left.2 \mathrm{~b}\right)$; the faster the IVR rate, the sooner the parking modes can participate in energy transport.

Very counter-intuitively though, the effects of the initially dominating contribution of the transporting mode and the delayed contribution of the parking modes exactly compensate each other so that $\left\langle x_{\text {site }}^{2}\right\rangle$ increases linearly in time. Hence, the total vibrational energy propagates in a diffusive manner, although the individual contributions of transporting and parking modes do not. The complexity of the IVR process within the sites, although it is rate limiting on these length and time scales, is completely hidden. The overall heat diffusivity $D$ is not influenced by the speed of intra-site IVR and has a constant value (see Appendix):

$$
D=\frac{c_{\text {trans }}}{c_{\text {total }}} k_{\mathrm{p}} \Delta x^{2}=k_{\mathrm{p}}^{\prime} \Delta x^{2}
$$

Hence, the overall process resembles diffusion on a simple 1D chain with an effective propagation rate $k_{\mathrm{p}}^{\prime}$ that equals the bulk value at all times. This effective rate $k_{\mathrm{p}}^{\prime}$ is given by the propagation rate $k_{\mathrm{p}}$, weighted with the ratio of the heat capacity of transporting versus total set of modes at a site $i, c_{\text {trans }} / c_{\text {total }}$ (the ratio is $1 / 4$ for the given model). The more parking modes are included in the individual sites of the chain, the less energy is available for propagation in the transporting modes. Intra-site IVR influences the population of the transporting modes, and thus the amount of vibrational energy that can be transported along the chain, but the transport speed depends only on $k_{\mathrm{p}}$ and 
not on $k_{\mathrm{IVR}}$. That is true even for very short times $\ll k_{\mathrm{IVR}}^{-1}$.

For simplicity, we had chosen in Figs. 1 and 2 a cascade-like connectivity of the localized states, but, as shown anlytically in the Appendix, Eq. 2 also holds for any more complex connectivity of parking modes including branches, etc.. Furthermore, we had assumed identical forward and backward rates between each two modes, which implicitly assumes an identical heat capacity of all modes. That is the case for low frequency, classical modes with $\hbar \omega<k_{B} T$, but not for higher-frequency modes. The quantummechanical heat capacity of higher frequency modes can be taken care of by adjusting the ratio of forward and backward rates accordingly, and again, as shown in the Appendix, this would not affect the result Eq. 2. However, the initial condition needs to be a state that is thermalized within the site that is initially heated (with a temperature that is higher than the rest of the chain). In our experiments, we observe essentially the same transport mechanism independent from the heating source [19-22]. One may therefore conclude that vibrational energy is not fed into the peptide chain in a mode-specific manner but is already randomized to a certain extent so that this condition is met reasonably well. That should be particularly true when heating through the plasmon resonance of a gold nanoparticle.

\section{Discussion}

The competing effects between parking and transporting modes cancel out when averaging equally over the energy content of all vibrational modes of one site. While all modes can easily be considered when analyzing a MD simulation (in fact, one commonly averages over all modes to achieve better statistics), the same is very difficult to implement in real experiment. Various fast microscopic "thermometers" have been used, like the SFG intensity of the symmetric $\mathrm{CH}_{3}$ stretching vibration [4], or the anti-Stokes Raman intensity of the $\mathrm{C}-\mathrm{Cl}[10]$ and position of the $\mathrm{C}=\mathrm{C}[41]$ 
vibrations. In our experiments [19-22], the amount of vibrational energy in the vicinity of a peptide unit is measured by probing the transient IR frequency shift of a localized $\mathrm{C}=\mathrm{O}$ mode. The same mechanism has been used in the context of other IR reporter groups like $\mathrm{C}-\mathrm{D}$ [6] and $\mathrm{NO}_{2}$-vibrations [7]. The physical origin of that thermometer is the well-known Dunham expansion of the vibrational energy

$$
E=\sum_{i} \hbar \omega_{i}\left(n_{i}+\frac{1}{2}\right)+\sum_{i j} x_{i j}\left(n_{i}+\frac{1}{2}\right)\left(n_{j}+\frac{1}{2}\right)
$$

where the $\omega_{i}$ are the harmonic frequencies of the various modes of a molecule, $n_{i}$ their occupation numbers, and $x_{i j}$ the anharmonic constants. The $\mathrm{C}=\mathrm{O}$ reporter mode itself will hardly be excited at the relevant temperatures because of its high frequency $\left(\approx 1650 \mathrm{~cm}^{-1}\right)$. Nevertheless, if lower-frequency modes get thermally excited, then the $\mathrm{C}=\mathrm{O}$ reporter mode will still experience a red-shift through the anharmonic coupling terms $x_{\mathrm{CO}, j}[23]$ :

$$
\Delta \omega_{\mathrm{CO}}=\sum_{j} x_{\mathrm{CO}, j} n_{j},
$$

In the experiment [19-22], we used this frequency shift as a measure of the amount of vibrational energy in the vicinity of the $\mathrm{C}=\mathrm{O}$ reporter mode.

Whereas transition frequencies can be determined with reasonable precision from quantum chemistry calculations, the calculation of anharmonic constants $x_{i j}$ is still very challenging [42]. Nevertheless, as shown by Leitner and coworkers, localized highfrequency modes preferably couple to other localized modes that overlap in space, while the anharmonic coupling between a localized and a delocalized mode is typically very small [39]. Therefore, one can assume that the local $\mathrm{C}=\mathrm{O}$ thermometer in our experiments [19-22] tentatively overestimates the contribution of other localized parking modes and does not sufficiently account for the energy in the delocalized transporting modes. In such a scenario, the measured apparent heat diffusivity is dominated by localized modes that receive vibrational energy delayed through intra-site 

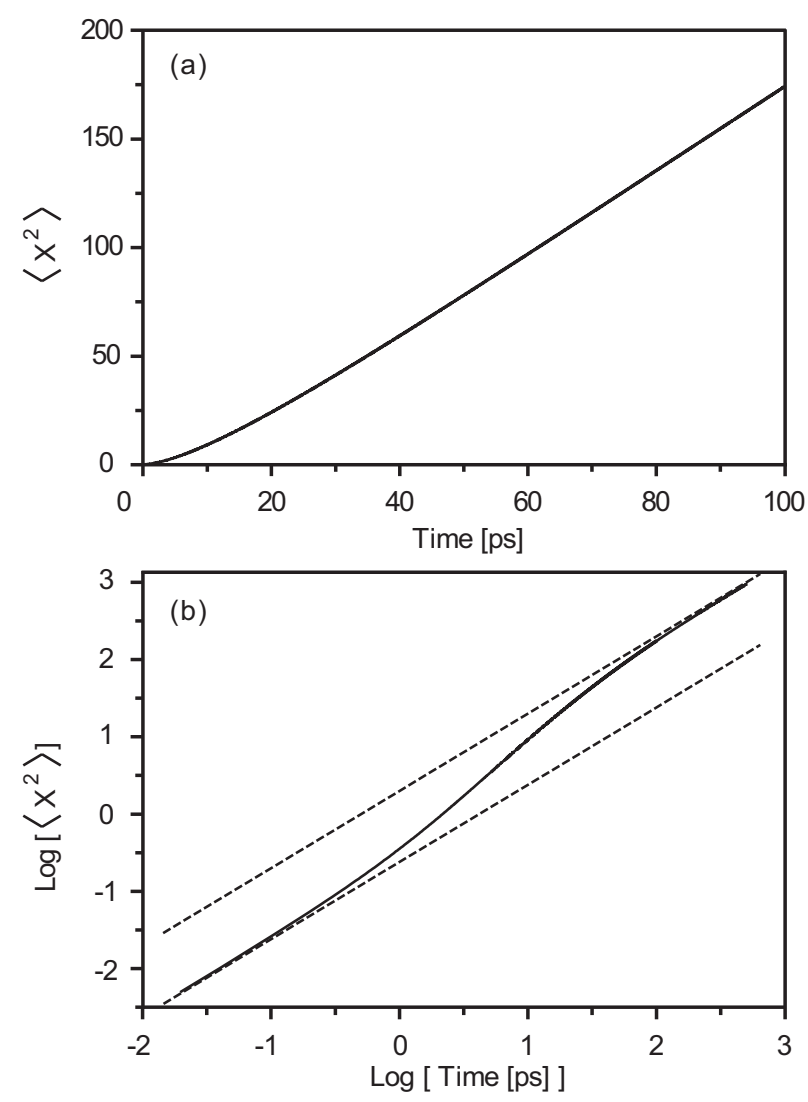

Fig. 3. Same calculation as in Fig. 2a, i.e., $k_{\mathrm{p}}=(0.2 \mathrm{ps})^{-1}$ and a fast IVR rate $k_{\mathrm{IVR}}=(10 \mathrm{ps})^{-1}$, but with the transporting modes underestimated by a factor of 10. (a) The mean square displacement of the energy of the entire site $\left\langle x_{\text {site }}^{2}\right\rangle$ plotted as a function of time now shows biphasic behavior. (b) The transition between two regimes with different heat diffusion constants $D$ can be nicely seen in a double logarithmic plot. Dashed lines with slope 1 are plotted to guide the eye.

IVR. Consequently, one underestimates the heat diffusivity in experiment.

To illustrate this effect, Fig. 3a shows the same simulation as Fig. 2a, but now with the contribution of the transporting modes underestimated by an arbitrary weighting factor of 10; i.e. assuming that the anharmonic constants $x_{\mathrm{CO}, j}$ between the $\mathrm{C}=\mathrm{O}$ reporter mode to transporting modes are in average 10 times smaller than to other parking modes. In contrast to Fig. 2, the measured heat diffusivity is no longer constant. The effect is most obvious in a double logarithmic plot of $\left\langle x_{\text {site }}^{2}\right\rangle$ versus $t$, which is shown in Fig. 3b. Two regimes can be identified, in both of which propagation is diffusive, indicated by a slope of 1 in the double 
logarithmic representation. The apparent heat diffusivity at early times is reduced by the weighting factor 10, and switches towards the bulk value on the timescale of intra-site IVR (10 ps).

This finding provides the missing puzzle piece for resolving why the MD simulations of the real peptide revealed a propagation rate that is about a factor of five faster than the experimentally measured value [19]. The reason is simply that the experimentally measured observable, i.e. the transient red-shift, does not reflect the overall residue energy, which, however, was the basis for all MD simulations. Based on these ideas, Stock and coworkers modified and reran their MD simulations of energy transport through the peptide [43]. Instead of simulating the total residue energy of the amino acid sites, they calculated the time evolution of the energy of the localized $\mathrm{C}=\mathrm{O}$ modes. The propagation rates obtained from the modified MD simulations are about one order of magnitude smaller, which agrees much better with the slow time evolution observed in the experiment.

In certain cases, energy transport has been shown to occur in a ballistic manner when IVR is suppressed $[20,4,44]$. This is probably in particular the case when the chain has a very simple repeat unit with only very few, high frequency intra-site modes $[4,44]$. Hence, propagation along the transporting modes is not necessarily diffusive on short length scales, as assumed here for simplicity. Nevertheless, in the presence of disorder, it will transfer into a diffusive process at some length-scale, and we assume that the transition we observe in Fig. 3b occurs in a regime where this is already the case, at least for solution phase system.

\section{Conclusion}

If vibrational energy within an individual repeat unit would remain thermalized at all times (i.e., in the limit of very fast $k_{\mathrm{IVR}}$ ), their entire time-dependent energy content could be extracted from any vibrational mode because all modes are equilibrated. 
On very fast time scales, however, intra-site IVR is rate limiting so that vibrational energy does not thermalize on the time scale of transport. When vibrational excess energy is transported along the peptide backbone, the fraction of vibrational energy, that happens to be in transporting modes, may propagate quickly through the chain. The higher-frequency localized modes, on the other hand, pick up energy later when they are populated by intra-site IVR from the transporting modes. Under such conditions, it becomes important which vibrational modes are used to estimate the residue energy of an amino acid, i.e., what "thermometer" is used.

Employing localized reporter groups as thermometer - which commonly are more sensitive to other spatially overlapping localized modes - reveal a heat diffusivity, which shows biphasic behavior. The initial transport of nonthermalized vibrational energy can be described by a reduced apparent heat diffusion constant. On longer time scales, heat transport can be described by the familiar bulk properties. The transition between both regimes occurs on a timescale determined by the rate of intrasite IVR. Hence, the low value we observe for the heat diffusivity at ultrafast timescales is not an artifact of a poor thermometer. It rather shows that averaging the vibrational energy content over entire amino acid residues oversimplifies the problem because it intrinsically assumes full thermalization of the individual sites at all times. This approach, which is commonly used in MD simulations, leads to the surprising result that all the complexity of IVR is completely hidden in a reduced, effective propagation rate.

\section{Appendix}

In the following, we show that if one measures the total vibrational energy of each site, one will see a diffusive process with the effects of intra-site IVR hidden, regardless how complex intra-site IVR is and despite the fact that it might actually be rate limiting. 
Let $\epsilon_{i, j}$ be the vibrational energy in mode $j$ of site $i$. Then, the kinetic equation can be written as (for an infinite chain)

$$
\begin{aligned}
\frac{d \epsilon_{i, j}}{d t}= & \delta_{j, 1}\left(k_{\mathrm{p}} \epsilon_{i-1,1}-2 k_{\mathrm{p}} \epsilon_{i, 1}+k_{\mathrm{p}} \epsilon_{i+1,1}\right) \\
& +\sum_{l} K_{j l}^{(\mathrm{IVR})} \epsilon_{i, l}
\end{aligned}
$$

where $\delta_{i, j}$ is the Kronecker symbol, $k_{\mathrm{p}}$ the propagation rate between sites, and $K_{j l}^{(\mathrm{IVR})}$ the relaxation matrix within each site, which is assumed to be the same for all sites. We furthermore assume energy conservation, $\sum_{j} K_{j l}^{(\mathrm{IVR})}=0$ for all $l$. Apart from that, the relaxation matrix can have any arbitrary form, i.e., can potentially be more complicated as the one indicated in Fig. 1, with any number of modes, branches, etc. If we calculate $\sum_{i} \epsilon_{i, j}$

$$
\sum_{i} \frac{d \epsilon_{i, j}}{d t}=\sum_{l} K_{j l}^{(\mathrm{IVR})}\left(\sum_{i} \epsilon_{i, l}\right)
$$

the first term of Eq. 5 cancels because of energy conservation. In the following, we assume we prepare an initial condition $\epsilon_{i, j}(t=$ 0 ) that is an equilibrium state of each site, but not necessarily an equilibrium state of the chain as a whole. That is, the temperature at one site might be elevated as compared to the other sites, but we assume that energy is thermalized within that one site. In other words, $\epsilon_{i, l}$ of each site $i$ is an eigenvector of matrix $K_{j l}^{(\mathrm{IVR})}$ with eigenvalue 0 . As such, also $\sum_{i} \epsilon_{i, l}$ is an eigenvector, and the rhs. of Eq. 6 is zero as well. We therefore find that

$$
\sum_{i} \epsilon_{i, j}(t)=\text { const }=\sum_{i} \epsilon_{i, j}(t=0) .
$$

If, on the other hand, we calculate $\sum_{j} \epsilon_{i, j}$

$$
\sum_{j} \frac{d \epsilon_{i, j}}{d t}=k_{\mathrm{p}} \epsilon_{i-1,1}-2 k_{\mathrm{p}} \epsilon_{i, 1}+k_{\mathrm{p}} \epsilon_{i+1,1}
$$

then the second term of Eq. 5 disappears, again because of energy conservation. 
Here, we pause, and first calculate the mean square displacement of a simple 1D chain (i.e., only one mode per site), whose kinetic equation is:

$$
\frac{d \epsilon_{i}}{d t}=k_{\mathrm{p}} \epsilon_{i-1}-2 k_{\mathrm{p}} \epsilon_{i}+k_{\mathrm{p}} \epsilon_{i+1}
$$

The mean square displacement is given as:

$$
\left\langle x^{2}\right\rangle=\Delta x^{2} \sum_{i} i^{2} \epsilon_{i}
$$

where $\Delta x$ is the separation of sites. We assume an initial excitation at site 0 with $\epsilon_{0}=1$, while all other sites are not populated at $t=0, \epsilon_{i}=0$ for $i \neq 0$. We obtain:

$$
\begin{aligned}
\frac{d}{d t}\left\langle x^{2}\right\rangle & =\Delta x^{2} \sum_{i} i^{2} \frac{d}{d t} \epsilon_{i} \\
& =k_{\mathrm{p}} \Delta x^{2} \sum_{i} i^{2}\left(\epsilon_{i-1}-2 \epsilon_{i}+\epsilon_{i+1}\right)
\end{aligned}
$$

Resorting the terms on the rhs., this simplifies to

$$
\frac{d}{d t}\left\langle x^{2}\right\rangle=2 k_{\mathrm{p}} \Delta x^{2} \sum_{i} \epsilon_{i}=2 k_{\mathrm{p}} \Delta x^{2}
$$

since we have energy conservation $\sum \epsilon_{i}=1$. Finally, we get

$$
\left\langle x^{2}\right\rangle=2 k_{\mathrm{p}} \Delta x^{2} t
$$

That is, the process is diffusive with a diffusion constant $D=$ $k_{\mathrm{p}} \Delta x^{2}$.

We now turn back to Eq. 8. As initial condition, we normalize the energy on site $0, \sum_{j} \epsilon_{0, j}=1$, and assume that all other sites are not populated, $\epsilon_{i, j}=0$ for $i \neq 0$. When one measures the total energy summed over all modes $j$ of a site $i, \epsilon_{i}^{\text {(tot) }}=\sum_{j} \epsilon_{i, j}$, the mean square displacement is given as:

$$
\left\langle x^{2}\right\rangle=\Delta x^{2} \sum_{i, j} i^{2} \epsilon_{i, j}
$$

We plug Eq. 8 into this expression, perform the same steps as in 
Eqs. 11 and 12, and obtain:

$$
\frac{d}{d t}\left\langle x^{2}\right\rangle=2 k_{\mathrm{p}} \Delta x^{2} \sum_{i} \epsilon_{i, 1}=2 k_{\mathrm{p}}^{\prime} \Delta x^{2}
$$

or

$$
\left\langle x^{2}\right\rangle=2 k_{\mathrm{p}}^{\prime} \Delta x^{2} t
$$

Here, we made use of the fact that $\sum_{i} \epsilon_{i, 1}=$ const $=\sum_{i} \epsilon_{i, 1}(t=$ $0)$ (Eq. 7). The effective propagation rate, and hence also the effective diffusion constant, is reduced by the relative energy of the transporting mode:

$$
k_{\mathrm{p}}^{\prime}=\epsilon_{0,1}(t=0) k_{\mathrm{p}}
$$

Since energy was assumed to be thermalized over all modes of site 0 at time 0 , the normalized energy $\epsilon$ is proportional to the heat capacity, and Eq. 2 is obtained. In summary, when the total energy $\epsilon_{i}^{(\text {tot })}$ is measured, then we observe a diffusive process with an effective propagation rate $k_{\mathrm{p}}^{\prime}$. All the complexity of the IVR process within the sites, which actually is rate limiting on short length and time scales, is completely hidden.

Note that Eq. 16 does not imply that Eq. 8 can be reduced to a true $1 \mathrm{D}$ chain with an effective propagation rate $k_{\mathrm{p}}^{\prime}$ :

$$
\frac{d \epsilon_{i}^{(\mathrm{tot})}}{d t}=k_{\mathrm{p}}^{\prime} \epsilon_{i-1}^{(\mathrm{tot})}-2 k_{\mathrm{p}}^{\prime} \epsilon_{i}^{(\mathrm{tot})}+k_{\mathrm{p}}^{\prime} \epsilon_{i}^{(\mathrm{tot})}
$$

Even though the mean square displacement predicted by Eqs. 8 and 18 shows the same time-dependence, the energies of the individual sites do not. Nevertheless, the differences between Eqs. 8 and 18 are small, in particular for sites not too close to the heating site, and might not be measurable in a real experiment.

Acknowledgment: We thank Gerhard Stock for many inspiring discussions on the topic. The work has been supported by an ERC advanced investigator grant (DYNALLO). 


\section{References}

[1] D. G. Cahill, W. K. Ford, K. E. Goodson, G. D. Mahan, A. Majumdar, H. J. Maris, R. Merlin, S. R. Phillpot, Nanoscale thermal transport, J. Appl. Phys. 93 (2003) 793-818.

[2] C. W. Chang, D. Okawa, A. Majumdar, A. Zettl, Solid-state thermal rectifier, Science 314 (2006) 1121-1124.

[3] J. Ren, P. Hänggi, B. Li, Berry-phase induced heat pumping and its impact on the fluctuation theorem, Phys. Rev. Lett. 104 (2010) 170601.

[4] Z. Wang, J. A. Carter, A. Lagutchev, Y. K. Koh, N. H. Seong, D. G. Cahill, D. D. Dlott, Ultrafast flash thermal conductance of molecular chains, Science 317 (2007) 787-790.

[5] D. Schwarzer, P. Kutne, C. Schröder, J. Troe, Intramolecular vibrational energy redistribution in bridged azulene-anthracene compounds: ballistic energy transport through molecular chains, J. Chem. Phys. 121 (2004) 1754-1764.

[6] S. R. G. Naraharisetty, V. M. Kasyanenko, J. Zimmermann, M. C. Thielges, F. E. Romesberg, I. V. Rubtsov, C-d modes of deuterated side chain of leucine as structural reporters via dual-frequency two-dimensional infrared spectroscopy, J. Phys. Chem. B 113 (2009) 4940-4946.

[7] K. Haiser, F. O. Koller, M. Huber, N. Regner, T. E. Schrader, W. J. Schreier, W. Zinth, Nitro-phenylalanine: a novel sensor for heat transfer in peptides, J. Phys. Chem. A 115 (2011) 2169-2175.

[8] Z. Wang, A. Pakoulev, D. D. Dlott, Watching vibrational energy transfer in liquids with atomic spatial resolution, Science 296 (2002) 2201-2203.

[9] D. Kuciauskas, C. J. Wohl, M. Pouy, A. Nasai, V. Gulbinas, Nonlinear optical spectroscopic studies of energy transfer in phospholipid bilayer liposomes embedded with porphyrin sensitizers, J. Phys. Chem. B 108 (2004) 1537615384.

[10] J. C. Deàk, Y. Pang, T. D. Sechler, Z. Wang, D. D. Dlott, Vibrational energy transfer across a reverse micelle surfactant layer, J. Phys. Chem. B 306 (2004) $473-476$.

[11] R. J. D. Miller, Vibrational energy relaxation and structural dynamics of heme proteins, Annu. Rev. Phys. Chem. 42 (1991) 581-614.

[12] T. Lian, B. Locke, Y. Kholodenko, R. M. Hochstrasser, Energy flow from solute to solvent probed by femtosecond ir spectroscopy: malachite green and heme protein solutions, J. Phys. Chem. 98 (1994) 11648-11656.

[13] P. Li, P. M. Champion, Investigations of the thermal response of laser-excited biomolecules, Biophys. J. 66 (1994) 430-436. 
[14] Y. Mizutani, T. Kitagawa, Direct observation of cooling of heme upon photodissociation of carbonmonoxy myoglobin, Science 278 (1997) 443-446.

[15] J. C. Owrutsky, D. Raftery, R. M. Hochstrasser, Vibrational relaxation dynamics in solutions, Annu. Rev. Phys. Chem. 45 (1994) 519-555.

[16] V. Kozich, W. Werncke, J. Dreyer, K. W. Brzezinka, M. Rini, A. Kummrow, T. Elsaesser, Vibrational excitation and energy redistribution after ultrafast internal conversion in 4-nitroaniline, J. Chem. Phys. 117 (2002) 719-726.

[17] T. Uzer, W. H. Miller, Theories of intramolecular vibrational-energy transfer, Phys. Rep. 199 (1991) 73-146.

[18] P. B. Graham, K. J. M. Matus, R. M. Stratt, The workings of a molecular thermometer: the vibrational excitation of carbon tetrachloride by a solvent, J. Chem. Phys. 121 (2004) 5348-5355.

[19] V. Botan, E. H. G. Backus, R. Pfister, A. Moretto, M. Crisma, C. Toniolo, P. H. Nguyen, G. Stock, P. Hamm, Energy transport in peptide helices, Proc. Natl. Acad. Sci. U.S.A. 104 (2007) 12749-12754.

[20] E. H. G. Backus, P. H. Nguyen, V. Botan, R. Pfister, A. Moretto, M. Crisma, C. Toniolo, O. Zerbe, G. Stock, P. Hamm, Structural flexibility of a helical peptide regulates vibrational energy transport properties, J. Phys. Chem. B 112 (2008) 15487-15492.

[21] M. Schade, A. Moretto, M. Crisma, C. Toniolo, P. Hamm, Vibrational energy transport in peptide helices after excitation of c-d modes in leu-d10, J. Phys. Chem. B 113 (2009) 13393-13397.

[22] M. Schade, A. Moretto, P. M. Donaldson, C. Toniolo, P. Hamm, Vibrational energy transport through a capping layer of appropriately designed peptide helices over gold nanoparticles, Nano Lett. (2010) 3057-3061.

[23] P. Hamm, S. M. Ohline, W. Zinth, Vibrational cooling after ultrafast photoisomerization of azobenzene measured by femtosecond infrared spectroscopy, J. Chem. Phys. 106 (1997) 519-529.

[24] D. R. Lide, CRC Handbook of chemistry and physics, 75 ed., CRC Press, Boca Raton FL, 1994.

[25] Y. P. Touloukian, R. W. Powelland, C. Y. Ho, M. C. Nicolaou, Thermal physical properties of matter, Plenum, New York, 1978.

[26] X. Yu, D. M. Leitner, Vibrational energy transfer and heat conduction in a protein, J. Phys. Chem. B 107 (2003) 1698-1707.

[27] X. Yu, D. M. Leitner, Heat flow in proteins: computation of thermal transport coefficients, J. Chem. Phys. 122 (2005) 054902.

[28] D. M. Leitner, Heat transport in molecules and reaction kinetics: the role of quantum energy flow and localization, in: Geometric structures of phase space in multidimensional chaos: a special volume of Adv. Chem. Phys., Part B, Volume 130, John Wiley \& Sons, Inc., 2005. 
[29] D. M. Leitner, Energy flow in proteins, Annu. Rev. Phys. Chem. 59 (2008) 233-259.

[30] E. R. Henry, W. A. Eaton, R. M. Hochstrasser, Molecular-dynamics simulations of cooling in laser-excited heme-proteins, Proc. Natl. Acad. Sci. U.S.A. 83 (1986) $8982-8986$.

[31] M. Tesch, K. Schulten, A simulated cooling process for proteins, Chem. Phys. Lett. 169 (1990) 97-102.

[32] K. Moritsugu, O. Miyashita, A. Kidera, Vibrational energy transfer in a protein molecule, Phys. Rev. Lett. 85 (2000) 3970-3973.

[33] I. Okazaki, Y. Hara, M. Nagaoka, On vibrational cooling upon photodissociation of carbonmonoxymyoglobin and its microscopic mechanism from the viewpoint of vibrational modes of heme, Chem. Phys. Lett. 337 (2001) 151-157.

[34] D. E. Sagnella, J. E. Straub, Directed energy "funneling" mechanism for heme cooling following ligand photolysis or direct excitation in solvated carbonmonoxy myoglobin, J. Phys. Chem. B 105 (2001) 7057-7063.

[35] H. Fujisaki, J. E. Straub, Vibrational energy relaxation in proteins, Proc. Natl. Acad. Sci. U.S.A. 102 (2005) 6726-6731.

[36] M. Schade, P. Hamm, Vibrational energy transport in the presence of intrasite vibrational energy redistribution, J. Chem. Phys. 131 (2009) 044511.

[37] S. Alexander, O. Entin-Wohlman, R. Orbach, Phonon-fracton anharmonic interactions: the thermal conductivity of amorphous materials, Phys. Rev. B 34 (1986) 2726-2734.

[38] J. Fabian, Decay of localized vibrational states in glasses: a one-dimensional example, Phys. Rev. B 55 (1997) R3328-R3331.

[39] D. M. Leitner, Vibrational energy transfer and heat conduction in a onedimensional glass, Phys. Rev. B 64 (2001) 094201.

[40] D. Segal, A. Nitzan, P. Hänggi, Thermal conductance through molecular wires, J. Chem. Phys. 119 (2003) 6840-6855.

[41] K. Iwata, H. Hamaguchi, Microscopic mechanism of solute-solvent energy dissipation probed by picosecond time-resolved raman spectroscopy, J. Phys. Chem. A 101 (1997) 632-637.

[42] M. Bounouar, C. Scheurer, Reducing the vibrational coupling network in nmethylacetamide as a model for ab initio infrared spectra computations of peptides, Chem. Phys. 323 (2006) 87-101.

[43] P. H. Nguyen, S. Park, G. Stock, Nonequilibrium molecular dynamics simulation of the energy transport through a peptide helix, J. Chem. Phys. 132 (2010) 025102 .

[44] Z. Lin, I. I I I $\quad$ V. Rubtsov, Constant-speed vibrational signaling along polyethyleneglycol chain up to 60 å distance, submitted. 